\title{
Dimensional behaviour of entropy and information
}

\author{
Sergey Bobkov*and Mokshay Madiman ${ }^{\dagger \ddagger}$
}

Note: A slightly condensed version of this paper is in press and will appear in the Comptes Rendus de l'Académies des Sciences Paris, Série I Mathematique, 2011.

\begin{abstract}
We develop an information-theoretic perspective on some questions in convex geometry, providing for instance a new equipartition property for log-concave probability measures, some Gaussian comparison results for log-concave measures, an entropic formulation of the hyperplane conjecture, and a new reverse entropy power inequality for log-concave measures analogous to V. Milman's reverse Brunn-Minkowski inequality.
\end{abstract}

\section{Introduction}

This Note announces some of the results obtained in [7, 8, 9. Given a random vector $X$ in $\mathbb{R}^{n}$ with density $f(x)$, the entropy power is defined by $\mathcal{N}(X)=e^{2 h(X) / n}$, where, with a common abuse of notation, we write $h(X)$ for the Shannon entropy $h(f):=-\int_{\mathbb{R}^{n}} f \log f$.

Theorem 1.1. If $X$ and $Y$ are independent random vectors in $\mathbb{R}^{n}$ with log-concave densities, there exist affine entropy-preserving maps $u_{i}: \mathbb{R}^{n} \rightarrow \mathbb{R}^{n}$ such that

$$
\mathcal{N}(\widetilde{X}+\widetilde{Y}) \leq C(\mathcal{N}(X)+\mathcal{N}(Y))
$$

where $\widetilde{X}=u_{1}(X), \widetilde{Y}=u_{2}(Y)$, and where $C$ is a universal constant.

Observe that the Shannon-Stam entropy power inequality [24] implies that $\mathcal{N}(\tilde{X}+\tilde{Y}) \geq$ $\mathcal{N}(X)+\mathcal{N}(Y)$ is always true. Thus Theorem 1.1 may be seen as a reverse entropy power inequality for log-concave measures. The proof of this assertion, outlined in Section 3, is based on a series of propositions introduced in Section 2 including V. Milman's result on the existence of $M$-ellipsoids. Specializing to uniform distributions on convex bodies, we show that Theorem 1.1 recovers Milman's reverse Brunn-Minkowski inequality [19]. One may also think of Theorem 1.1 as completing the usual analogy between the Brunn-Minkowski and entropy power inequalities (see, e.g., [14]).

This Note is in the nature of a sketch meant as an entry point for the interested reader into the recent work by the authors in [7, 8, 9]. Therefore, details are kept to a minimum. However,

${ }^{*}$ S. G. Bobkov is with the School of Mathematics, University of Minnesota, 127 Vincent Hall, 206 Church St. S.E., Minneapolis, MN 55455 USA. Email: bobkov@math.umn.edu.

${ }^{\dagger}$ M. M. Madiman is with the Department of Statistics, Yale University, James Dwight Dana House, 24 Hillhouse Ave, New Haven, CT 06511 USA. Tel.: +1-203-432-0639. Fax: +1-203-432-0633. Email: mokshay.madiman@yale.edu.

${ }^{\ddagger}$ S.B. was supported in part by U.S. National Science Foundation grant DMS 0706866, and M.M. was supported in part by a Junior Faculty Fellowship from Yale University. 
it is appropriate to mention that the tools relied upon in our proof of Theorem 1.1 and allied results (some of which are not detailed in the sequel) include the localization lemma of Lovász-Simonovits [17, reverse Hölder type inequalities such as Berwald's inequality [4, 10, the studies of the hyperplane conjecture by Bourgain [13], Milman-Pajor [22], and Ball [1, 2], a submodularity property for the entropy of sums developed by the second-named author [18, and ideas from the literature on convex measures and isoperimetry developed by Borell [11, 12] and the first-named author [5, 6].

\section{Intermediate results}

\subsection{An equipartition property}

Let $X$ be a random vector taking values in $\mathbb{R}^{n}$, and suppose its distribution has a density $f$ with respect to Lebesgue measure on $\mathbb{R}^{n}$. The random variable $\widetilde{h}(X)=-\log f(X)$ may be thought of as the "information content" of $X$. Note that the entropy is $h(X)=\mathbf{E} \widetilde{h}(X)$.

Because of the relevance of the information content in information theory, probability, and statistics, it is intrinsically interesting to understand its behavior. In particular, a natural question arises: Is it true that the information content concentrates around the entropy in high dimension? In general, there is no reason for such a concentration property to hold. However, the following proposition shows that in fact, such a property holds uniformly for the entire class of log-concave densities.

Theorem 2.1. If $X$ has a log-concave density $f$ on $\mathbb{R}^{n}$, then for $0 \leq \varepsilon \leq 2$,

$$
\mathbf{P}\left\{\left|\frac{\widetilde{h}(X)}{n}-\frac{h(X)}{n}\right| \geq \varepsilon\right\} \leq 4 e^{-\varepsilon^{2} n / 16} .
$$

No normalization whatsoever is required for this result, which is proved in [7] using the localization lemma of Lovász-Simonovits, and certain reverse Hölder type inequalities for log-concave measures.

Equivalently, with high probability, $f(x)^{2 / n}$ is very close to the entropy power $N(X)=$ $\exp \left\{\frac{2}{n} h(X)\right\}$, and the distribution of $X$ itself is effectively the uniform distribution on the class of typical observables, or the "typical set" (defined to be the collection of all points $x \in \mathbb{R}^{n}$ such that $f(x)$ lies between $e^{-h(X)-n \varepsilon}$ and $e^{-h(X)+n \varepsilon}$, for some small fixed $\varepsilon>0$ ). The effective uniformity of the distribution of $X$ on some compact set, entailed by this concentration result, may be seen as an extension of the asymptotic equipartition property (or Shannon-McMillan-Breiman theorem) to non-stationary stochastic processes with logconcave marginals (cf. [7]).

If one is more interested in the effective support rather than an effective uniformity, one can simply consider a superlevel set (necessarily convex and compact) of the density $f$ instead of the annular region above. This effective support on a convex set implied by Theorem 2.1 allows (see [9]) the transference of some results from the setting of convex bodies to that of log-concave measures, in particular, the existence of $M$-ellipsoids [19, 21, 20, 23]. (Such a transference technique based on looking at superlevel sets of log-concave densities has been anticipated before, e.g., by [16], but Theorem 2.1 refines those observations and identifies the underlying concentration phenomenon.)

Corollary 2.2. Let $\mu$ be a probability measure on $\mathbf{R}^{n}$ with log-concave density $f$ such that $\|f\|_{\infty} \geq 1$ (where $\|f\|_{\infty}$ is the essential supremum and hence the maximum of $f$ ). Then 
there exists an ellipsoid $\mathcal{E}$ of volume 1 such that $\mu(\mathcal{E})^{1 / n} \geq c_{M}$ for some universal constant $c_{M} \in(0,1)$.

Equivalently, for some linear volume-preserving map $u: \mathbf{R}^{n} \rightarrow \mathbf{R}^{n}, \mu u^{-1}(D)^{1 / n} \geq c_{M}$, where $D$ is the Euclidean ball of volume one.

\subsection{Entropy and the maximal density value}

Trivially $h(X) \geq \log \|f\|_{\infty}^{-1}$. In fact, one can also bound the entropy from above using the maximal density value under log-concavity (see [8]).

Theorem 2.3. If a random vector $X$ in $\mathbf{R}^{n}$ has log-concave density $f$, then

$$
\log \|f\|_{\infty}^{-1 / n} \leq \frac{1}{n} h(X) \leq 1+\log \|f\|_{\infty}^{-1 / n}
$$

The hyperplane conjecture or slicing problem (cf. Bourgain [13] or Ball [2]) asserts that there exists a universal, positive constant $c$ (not depending on $n$ ) such that for any convex set $K$ of unit volume in $\mathbb{R}^{n}$, there exists a hyperplane $H$ passing through its centroid such that the $(n-1)$-dimensional volume of the section $K \cap H$ is bounded below by $c$. There are several equivalent formulations of the conjecture, all of a geometric or functional analytic flavor (even the ones that nominally use probability). The current best bound known, due to Klartag [15], is $\Omega\left(n^{-1 / 4}\right)$. Theorem 2.3 gives a purely information-theoretic formulation of the hyperplane conjecture. For a random vector $X$ in $\mathbb{R}^{n}$ with density $f$, let $D(X)$ or $D(f)$ denote its relative entropy from Gaussianity (which is the relative entropy from the Gaussian $g$ with the same mean and covariance matrix, and also equals the difference $h(g)-h(f)$ ). The Entropic Form of the Hyperplane Conjecture asserts that for any log-concave density $f$ on $\mathbb{R}^{n}, D(f) \leq c n$ for some universal constant $c$. It is easy to see then that another equivalent form of the hyperplane conjecture is that the entropic distance from independence (i.e., the relative entropy of any log-concave measure from the product of its marginals) is also bounded by $c n$ for some universal constant $c$. As an aside, Klartag's result combined with our equivalence implies that $D(f) \leq \frac{1}{4} n \log n+c n$ for any log-concave $f$. This is already the first quantitative demonstration of the spiritual closeness of log-concave measures to Gaussians, which has been observed in qualitative ways numerous times (e.g., behavior as regards functional inequalities). Let us note en passant that entropy plays a role in Ball's [3] proof that the KLS conjecture implies the hyperplane conjecture.

\section{Proof outline of Theorem 1.1}

The following "submodularity" property of the entropy functional with respect to convolutions was obtained in [18]: Given independent random vectors $X, Y, Z$ in $\mathbf{R}^{n}$ with absolutely continuous distributions, we have

$$
h(X+Y+Z)+h(Z) \leq h(X+Z)+h(Y+Z)
$$

provided that all entropies are well-defined.

Let $Z \sim \operatorname{Unif}(D)$, where $D$ is the centered Euclidean ball with volume one. Since $h(Z)=$ 0 , the submodularity property implies

$$
h(X+Y) \leq h(X+Y+Z) \leq h(X+Z)+h(Y+Z),
$$


for random vectors $X$ and $Y$ in $\mathbf{R}^{n}$ independent of each other and of $Z$.

Let $X$ and $Y$ have log-concave densities. Due to homogeneity of Theorem 1.1, assume without loss of generality that $\|f\|_{\infty} \geq 1$ and $\|g\|_{\infty} \geq 1$. Then, our task reduces to showing that both $\mathcal{N}(X+Z)$ and $\mathcal{N}(Y+Z)$ can be bounded from above by universal constants.

By Corollary 2.2, for some affine volume preserving map $u: \mathbf{R}^{n} \rightarrow \mathbf{R}^{n}$, the distribution $\widetilde{\mu}$ of $\widetilde{X}=u(X)$ satisfies $\widetilde{\mu}(D)^{1 / n} \geq c_{M}$ with a universal constant $c_{M}>0$. Let $\tilde{f}$ denote the density of $\widetilde{X}=u(X)$. Then the density $p$ of $S=\widetilde{X}+Z$, given by $p(x)=\int_{D} \tilde{f}(x-z) d z=$ $\widetilde{\mu}(D-x)$, satisfies $\|p\| \geq p(0) \geq c_{M}^{n}$. Applying Theorem 2.3 to the random vector $S$, $\mathcal{N}(S) \leq C\|p\|_{\infty}^{-2 / n} \leq C \cdot c_{M}^{-2}$, which completes the proof.

Remark 1. Recall C. Borell's hierarchy of convex measures on $\mathbf{R}^{n}$, classified by a parameter $\kappa \in[-\infty, 1 / n]$. In this hierarchy, $\kappa=0$ corresponds to the class of log-concave measures. When $\kappa>0$, a $\kappa$-concave probability measure is necessarily compactly supported on some convex set.

For any random vector $X$ with values in $A$, there is a general upper bound $h(X) \leq \log |A|$. Using Berwald's inequality, we provide a complementary estimate from below depending only on the "strength" of convexity of the density $f$ of $X$ : Let $X$ be a random vector in $\mathbf{R}^{n}$ having an absolutely continuous $\kappa$-concave distribution supported on a convex body $A$ with $0<\kappa \leq 1 / n$. Then $h(X) \geq \log |A|+n \log (\kappa n)$. Note when $\kappa=1 / n$, this bound is sharp.

Assume a probability measure $\mu$ is $\kappa^{\prime}$-concave on $\mathbf{R}^{n}$ and a probability measure $\nu$ is $\kappa^{\prime \prime}$-concave on $\mathbf{R}^{n}$. If $\kappa^{\prime}, \kappa^{\prime \prime} \in[-1,1]$ satisfy

$$
\kappa^{\prime}+\kappa^{\prime \prime}>0, \quad \frac{1}{\kappa}=\frac{1}{\kappa^{\prime}}+\frac{1}{\kappa^{\prime \prime}},
$$

then their convolution $\mu * \nu$ is $\kappa$-concave. Hence, if random vectors $X_{1}$ and $X_{2}$ are independent and uniformly distributed in convex bodies $A_{1}$ and $A_{2}$ in $\mathbf{R}^{n}$, then the sum $X_{1}+X_{2}$ has a $\frac{1}{2 n}$-concave distribution supported on the convex body $A_{1}+A_{2}$. The preceding entropy bound then implies that $h\left(X_{1}+X_{2}\right) \geq \log \left|A_{1}+A_{2}\right|-n \log 2$. This immediately allows one to deduce Milman's reverse Brunn-Minkowski inequality from Theorem 1.1.

Remark 2. Theorems 1.1 and 2.3 have been extended to the larger class of convex measures [9, 8.

Remark 3. It is instructive to write some of the above observations as entropy comparison results. For example, the bound in Remark 1 says that if $f$ is a density of the random vector $X$ taking values in the convex body $A$, and if $\mathrm{U}_{A}$ is the uniform distribution on $A$,

$$
0 \leq \frac{1}{n} D\left(f \| \mathrm{U}_{A}\right)=\frac{1}{n} h\left(\mathrm{U}_{A}\right)-\frac{1}{n} h(X) \leq C .
$$

where $D(f \| g)=\int f(x) \log \frac{f(x)}{g(x)} d x$ is the relative entropy. In this form, the constant upper bound on the difference between relative entropies per coordinate expresses a dimension-free approximation of the distribution of $X$ by the uniform distribution $\mathrm{U}_{A}$. On the other hand, Theorem 2.3 can be rewritten as saying that if $Z$ in $\mathbb{R}^{n}$ is any normally distributed random vector with maximum density being the same as that of $X$, then

$$
\frac{1}{n} h(Z)-\frac{1}{2} \leq \frac{1}{n} h(X) \leq \frac{1}{n} h(Z)+\frac{1}{2} .
$$

This is a dimension-free approximation of the entropy of $X$ by a Gaussian entropy. The entropic formulation of the hyperplane conjecture described in Section 2 is another such dimension-free approximation, both of entropy and distributional (see [8] for details). 


\section{References}

[1] K. Ball. Isometric problems in $\ell^{p}$ and sections of convex sets. $\mathrm{PhD}$ thesis, University of Cambridge, UK, 1986.

[2] K. Ball. Logarithmically concave functions and sections of convex sets in $\mathbf{R}^{n}$. Studia Math., 88(1):69-84, 1988.

[3] K. M. Ball. Information decrease along semigroups. Talk given at conference on Banach Spaces and Convex Geometric Analysis, Universität Kiel, Germany, April 2003.

[4] L. Berwald. Verallgemeinerung eines Mittelwertsatzes von J. Favard für positive konkave Funktionen. Acta Math., 79:17-37, 1947.

[5] S. G. Bobkov. Large deviations and isoperimetry over convex probability measures with heavy tails. Electron. J. Probab., 12:1072-1100 (electronic), 2007.

[6] S. G. Bobkov. Convex bodies and norms associated to convex measures. Probab. Theory Related Fields, 147(1-2):303-332, 2010.

[7] S. G. Bobkov and M. Madiman. Concentration of the information in data with logconcave distributions. Ann. Probab., in press, arXiv:1012.5457 [math.PR], 2010.

[8] S. G. Bobkov and M. Madiman. The entropy per coordinate of a random vector is highly constrained under convexity conditions. arXiv:1006:2883 [math.PR], 2010.

[9] S. G. Bobkov and M. Madiman. Reverse Brunn-Minkowski and reverse entropy power inequalities for convex measures. Preprint, 2010.

[10] C. Borell. Complements of Lyapunov's inequality. Math. Ann., 205:323-331, 1973.

[11] C. Borell. Convex measures on locally convex spaces. Ark. Mat., 12:239-252, 1974.

[12] C. Borell. Convex set functions in d-space. Period. Math. Hungar., 6(2):111-136, 1975.

[13] J. Bourgain. On high-dimensional maximal functions associated to convex bodies. Amer. J. Math., 108(6):1467-1476, 1986.

[14] A. Dembo, T.M. Cover, and J.A. Thomas. Information-theoretic inequalities. IEEE Trans. Inform. Theory, 37(6):1501-1518, 1991.

[15] B. Klartag. On convex perturbations with a bounded isotropic constant. Geom. Funct. Anal., 16(6):1274-1290, 2006.

[16] B. Klartag and V. D. Milman. Geometry of log-concave functions and measures. Geom. Dedicata, 112:169-182, 2005.

[17] L. Lovász and M. Simonovits. Random walks in a convex body and an improved volume algorithm. Random Structures Algorithms, 4(4):359-412, 1993.

[18] M. Madiman. On the entropy of sums. In Proc. IEEE Inform. Theory Workshop, pages 303-307. Porto, Portugal, 2008.

[19] V. D. Milman. Inégalité de Brunn-Minkowski inverse et applications à la théorie locale des espaces normés. C. R. Acad. Sci. Paris Sér. I Math., 302(1):25-28, 1986. 
[20] V. D. Milman. Entropy point of view on some geometric inequalities. C. R. Acad. Sci. Paris Sér. I Math., 306(14):611-615, 1988.

[21] V. D. Milman. Isomorphic symmetrizations and geometric inequalities. In Geometric aspects of functional analysis (1986/87), volume 1317 of Lecture Notes in Math., pages 107-131. Springer, Berlin, 1988.

[22] V. D. Milman and A. Pajor. Isotropic position and inertia ellipsoids and zonoids of the unit ball of a normed $n$-dimensional space. In Geometric aspects of functional analysis (1987-88), volume 1376 of Lecture Notes in Math., pages 64-104. Springer, Berlin, 1989.

[23] G. Pisier. The volume of convex bodies and Banach space geometry, volume 94 of Cambridge Tracts in Mathematics. Cambridge University Press, Cambridge, 1989.

[24] A.J. Stam. Some inequalities satisfied by the quantities of information of Fisher and Shannon. Information and Control, 2:101-112, 1959. 